\title{
Strategy-as-practice: The construction of university large instruments sharing platform
}

\author{
Jun Xiao \\ School of Mechanical and Electronic Engineering, Wuhan University of Technology, Wuhan \\ 430070, China
}

Keywords: Large instrument, Sharing platform, Strategy-as-practice

\begin{abstract}
In recent years, with the state fund of higher education increasing, the amount of university large instruments has been increased significantly. However, the low utilization rates of large instruments are becoming more and more serious. This paper analyzes the problems existing in instruments sharing, as well as explores the measures and suggestions in the construction of large instrument sharing platform from the aspects of demonstrating the feasibility of purchasing, so as to improve the management system and the operation mechanism and thereby extend the scope of strategy-as-practice of university large instruments sharing platform.
\end{abstract}

\section{Introduction}

In recent years, with growing of the overall strength and improving of the teaching and researching conditions in university, there's been a sharp rise in the total amount of university large instruments. However, the utilization rate of large instruments is not high for various reasons. Large instruments are the technical foundation and important means to break through the frontier of science and to solve the problems of social development and national security ${ }^{[1]}$. So colleges and universities are faced with the ticklish problem that how to increase the utilization ratio and the use efficiency of large instruments.

\section{The problems of the sharing instrument}

Science and engineering universities' instrument have the characteristics of large number and different classification. Because of this, they are the main force of sharing instrument. With practical experience, the paper analyses the problems of the sharing instrument. The reasons that the utilization ratio and the use efficiency of large instruments are low are listed as follows:

(1) Lacking of professional personnel

Most large instruments are precision instrument, which requires high-quality professionals to operate and maintain avoiding damage. However, in practice, the personnel training of laboratory often lags behind the laboratory construction due to various reasons. Because of this, many large instruments without professional management resulting in lay idle ${ }^{[2]}$.

(2) Lacking of financial security

The universities invest heavily in purchasing instruments, but the new revenue to pay for operation and maintenance is scarcely. Because of the effective maintenance can not to be provided, the lives of the instruments have been greatly reduced ${ }^{[3]}$.

(3) Lacking of the matching system as the guarantee

Now many universities have established large instrument sharing platform, but they are biased towards quantity rather than quality of sharing. To a large extent, the construction of sharing platform is lacking effective management, evaluation, assessment and incentive mechanism.

(4) Lacking of the awareness of sharing.

Some units, laboratories, research group consider the instruments are private, not willing to share. They think the sharing of instrument will affect its own productivity, which go against the teaching and scientific research ${ }^{[4]}[5]$. 


\section{The idea of how to construct large instruments sharing platform.}

\subsection{Should stress demonstrate the feasibility of purchasing to avoid repeat purchase the large in strument}

Formulating the detailed rules and standard process for demonstrate of the feasibility of purchasing large instruments, so as to make the demonstration work more standardization and institutionalization. Checking the source to avoid repeat purchase of large instruments. Where the budget unit price more than 100 thousand yuan or budget total price more than 200 thousand yuan, feasibility argumentation is needed regardless of the source of funding.

(1) Comprehensive evaluation for necessity, advancement, applicability, rationality and sharing of the intended purchasing instruments.

The main content include: Implementation of the funds of purchase and then annual maintenance; Staffing of management and maintenance for the large instruments; other requirements just like installed site, auxiliary facilities, environment and so on; The similar instrument allocation status of province; The sharing scheme of inside and outside school; Forecast the using benefit of instrument and risk analysis.

(2) According to the different price ranges of the budget, hierarchical organize demonstrate the feasibility of purchasing

Where the budget unit price or budget total price 100-400 thousand yuan, feasibility argumentation is organized by the project team; Where the budget unit price or budget total price 400-1200 thousand yuan, feasibility argumentation is organized by the uint; Where the budget unit price or budget total price more than 1.2 million yuan, feasibility argumentation is organized by the state-owned assets management department and the unit.

(3) The feasibility argumentations have been taken in the form of expert demonstration meeting. This panel shall consist of three members: academic expert, technical expert and management expert.

(4) The team leaders chair the expert demonstration meeting. The main process for meeting including unit made feasibility analysis report, experts questioned (on-site inspection when necessary), expert discussion, expert group formed opinions, expert autograph.

\subsection{Strengthening construction of sharing platform to promote the state-owned assets management level.}

Formulated the interim measures on large instrument sharing management and the interim measures on large instrument using performance appraisal management, to enhance the level of large instruments open sharing and promote large instrument providing service facing the society.

(1) Finish building information management platform and instrument open sharing system. The school to construction the sharing platform by policy guiding, insist on multi-construction and sharing. We construct "University - Institute - Research Group "three-level platform management system, which under the principle of "top-level design, overall planning, relying on discipline, relatively centralized, open sharing, and professional service".

(2) Strengthen capital investment and integration of resources. School has invested 2.7 million yuan in three successive years, and apply for funds 200 thousand yuan from education department' CERS [6]. The school level large instrument sharing service platform has been completed in three phases. The whole system is divided into school level and college level two cascades.

(3)Improve platform management and operation mechanism. The Schools' investment management pattern to implement two-stage management, and strengthen the institutional building of the assessment, incentives, constraints, security and training.

(4) Strengthen the evaluating on benefit of large instruments. An assessment team has been set up in the school to overall responsibility for assessment work of all levels of large instrument platform. Assessment results were taken as important reference for adjustment of people positions and annual bonus, and also have influenced the budget of large instruments purchasing. 


\section{Construction effect of large instrument sharing platform.}

We have built the platform in 14 departments, established 92 open laboratories and 175 monitors, at the same time we have brought 320 instruments into sharing platform by the internet of things technology in the past three years. The economic and social benefit, instrument utilization ratio are improved significantly by opening and sharing large instrument.

By sharing platform, we realize great-leap-forward development of technological innovation. We have creative brought overall flow of metrology accreditation management system into overall flow to platform reservation, testing, pricing and downloading data. We are the first who truly realized overall flow of metrology accreditation system network, digitalization and standardization management, so significantly improving service efficiency of open sharing.

\section{Conclusion}

The construction of large instrument sharing platform is a systematic project, which requires longterm investment, continuous exploration, innovation, research, and improve the management system. We should constantly stress demonstrate the feasibility of purchasing, so as to avoid repeat purchase and leave unused of the large instrument. To better realize the national interests, we must continuously improve management efficiency of large instrument, by measures such as appraise the use efficiency of large instrument, perfect the function of sharing platform, and achieve information of instrument management.

\section{References}

[1]. No. [2014]70, the opinions of the State Council on the opening of the national major scientific research infrastructure and large-scale scientific research instruments to the public

[2]. Weifeng Ying, Yan GAO, Huiwu Zhao. Research on some problems of open sharing of large scale instruments and equipment in Colleges and universities [J]. Experimental techniques and management, 2011, 28 (11): 213-216

[3]. Hong Zeng. Discussion on sharing mechanism and management mode of large scale instruments and equipment in Colleges and universities [J]. Modern educational science, 2006 (11): 109-111

[4]. Fang $\mathrm{Hu}, \mathrm{Hao} \mathrm{Xu}$. Discussion on the construction of large scale precision instrument sharing platform in Colleges and universities [J]. Laboratory research and exploration, 2007, 26 (2): 140142

[5]. Jingkui Li, et al. The practice and exploration of the construction of instrument and equipment sharing platform in Colleges and universities [J]. Laboratory research and exploration, 2012, 31 (4): $452-454$

[6]. Zhaoyang Fan. Thoughts and Practice on the construction of open sharing platform for large scale instruments and equipment in Colleges and universities [J]. Experimental technology and management, 2011, 28 (10): 194-197 